Original article

\title{
The impact of sleep duration and sleep quality on glycaemic control in Asian population with type 2 diabetes mellitus: A systematic literature review and meta-analysis of observational studies
}

\author{
Md Azharuddin ${ }^{\mathrm{a}}$, Prem Kapur ${ }^{\mathrm{b}}$, Mohammad Adil ${ }^{\mathrm{c}}$, Pinaki Ghosh ${ }^{\mathrm{d}}$, Manju Sharma ${ }^{\mathrm{c}, *}$ \\ ${ }^{a}$ Department of Pharmaceutical Medicine, Division of Pharmacology, School of Pharmaceutical Education and Research, Jamia Hamdard, New Delhi, 110062, India \\ ${ }^{\mathrm{b}}$ Department of Medicine, Hamdard Institute of Medical Sciences and Research, Jamia Hamdard, New Delhi, 110062, India \\ ${ }^{\mathrm{c}}$ Department of Pharmacology, School of Pharmaceutical Education and Research, Jamia Hamdard, New Delhi, 110062, India \\ ${ }^{\mathrm{d}}$ Department of Pharmacology, Poona College of Pharmacy, Bharati Vidyapeeth, Pune, 411038, India
}

\section{A R T I C L E I N F O}

\section{Keywords:}

Sleep duration

Sleep quality

Glycaemic control

Asian population

Type 2 diabetes mellitus

Meta-analysis

\begin{abstract}
A B S T R A C T
Aim: Excessive and insufficient sleep disrupts glycaemic control in patients with type 2 diabetes mellitus (T2DM). However, the evidence underlying the association between sleep duration, sleep quality and glycaemic control in Asian diabetic population remains unclear. We aimed to perform a systematic literature review and meta-analysis of observational studies to examine the sleep duration and sleep quality on glycaemic control in Asian population with T2DM.

Methods: A systematic search was performed on PubMed and Embase from inception through April 2019 to identify studies assessing the relationship between sleep duration, sleep quality and glycemic control in Asian population with T2DM. Estimated the pooled mean difference (MD) and 95\% confidence intervals (CIs) using a weighted random-effect model.

Results: Overall, seven studies were included in the meta-analysis. The results suggesting short sleep was associated with significantly higher HbA1c levels compared to normal sleep (MD: 0.11; 95\% CI: 0.06-0.17). While long sleep duration was associated with significantly higher fasting plasma glucose levels (FPG) (MD: 5.30; 95\% CI: 3.27-7.34) compared to normal sleep duration. Good sleep quality was significantly reduced the FPG levels (MD: 11.28; 95\% CI: 5.13-17.42), compared to poor sleep quality.

Conclusions: Our study suggested that sleep duration, as well as the quality of sleep, is thought to be an important factor in the metabolic function of type 2 diabetes patients. Further studies warrant to confirm the present findings and to establish the potential causal relationship between sleep and glycaemic control.
\end{abstract}

\section{Introduction}

Globally diabetes mellitus (DM) has become a major public health concern and contributed leading cause of death in most countries. ${ }^{1}$ According to the International Diabetes Federation (IDF), the number of diabetic population worldwide is estimated more than 425 million people, and it may predict to rise up to 629 million by 2045 . the epidemiological burden is increasing markedly in the people live in low and middle income countries. According to the IDF 2017 South-East Asia and Western Pacific regions are became the epicenter of the diabetes crisis, China alone contributing 121 million people suffering with diabetes and India accounted for a total of 74 million of population with diabetes population. ${ }^{2}$ Diabetes is a major risk contributor for cardiovascular disease (CVD), which is the common leading cause of death in diabetic patients. ${ }^{3}$ Glycemic control is a key factor in reducing a cardiovascular risk in diabetic patients. With increasing risk of CVD among diabetic subjects, it is crucial to identify modifiable lifestyle factors associated to lowering the risk of metabolic syndrome. Sleep health is a one of the novel and imperative modifiable risk factor for better glycaemic control in patients with T2DM. ${ }^{4}$

Worldwide sleep disturbances and deprivation has become more prevalent over the recent years, and associated with the significant burden of T2DM and obesity. According to the data of National Sleep Foundation's 2018 suggested that $46 \%$ of American adults having poor sleep, and $10 \%$ of American adults prioritize their sleep for betterment of health status over other aspects of daily living such as fitness/

\footnotetext{
Abbreviation: IV, inverse variance; HbA1c, glycated haemoglogin; FPG, fasting plasma glucose

* Corresponding author.

E-mail address: msharma@jamiahamdard.ac.in (M. Sharma).
} 
nutrition, work, social life, and hobbies/personal interests. ${ }^{5}$

Several meta-analysis accounted the impact of sleep duration and sleep quality on health outcomes. These studies reported the relationship between sleep duration and obesity, ${ }^{6}$ hypertension, ${ }^{7}$ type 2 diabetes, ${ }^{8}$ cardiovascular outcomes (including coronary heart disease, stroke and total CVD, ${ }^{9}$ and all-cause mortality. ${ }^{10}$

Inadequate sleep could affect metabolic function, ${ }^{11}$ endocrine systems ${ }^{12}$ as well as immune pathway. ${ }^{13}$

In recent, the evidence from meta-analysis which assess the duration and quality of sleep also suggests that amount of sleep and quality of sleep are significantly play an important role in metabolic function in patients with type 2 diabetes mellitus ${ }^{4}$ and predictors of the development of type 2 diabetes. ${ }^{8}$ Long sleep duration or short sleep duration, and irregular sleep wake patterns significantly associated with higher BMI and impaired glycemic control. ${ }^{14}$

The overall data of these studies indicated that sleep duration and quality of sleep might be associated with increased risk of metabolic syndrome, CVD morbidity and mortality.

Beyond the impact and association of short or long sleep duration and poor sleep quality, it makes detrimental effect on health related quality of life as well. Excessive and insufficient sleep disrupts glycaemic control in patients with T2DM. However, there is lack of evidence on the association between sleep duration, sleep quality and glycaemic control in Asian population with T2DM. Therefore, we sought to perform a systematic literature review and meta-analysis of observational studies to provide more robust evidence regarding the association between sleep duration, sleep quality and glycemic control in T2DM patients.

\section{Materials and methods}

The current meta-analysis was performed according to the guideline of the PRISMA (Preferred Reporting Items for Systematic Reviews and Meta-analyses). ${ }^{15}$

\subsection{Search strategy}

We performed a systematic search on PubMed, and Embase from inception through April 2019 to identify relevant published studies that assess the relationship between sleep duration, sleep quality and glycemic control in Asian population with T2DM.

The search strategy used the following text words or Medical Subject Headings (Mesh): "type 2 diabetes", "type 2 diabetes mellitus", "T2DM", "sleep", "sleep duration", "sleep quality". Manual searches of references were also performed on Google Scholar and bibliographies of included studies and previous reviews were also examined to identify additional eligible publications. The literature search was performed with no time-frame restrictions. Language other than English was not included in the analysis.

\subsection{Study selection}

Study selection was based on the initial screen of identified titles and abstracts and the second screen of full-text articles. Studies were considered eligible if they met the following inclusion criteria: (1) patients with T2DM; (2) the exposure of interest was, studies examined the sleep quality and association between sleep quality and patients with T2DM, where sleep quality was examined by either an explicit questionnaire; (3) duration of sleep in hours or sleep quantity; (4) the outcome of interest was HbA1c (glycated haemoglobin) levels (test for glycaemic control in T2DM) and other outcome was fasting plasma glucose (FPG) levels; (5) the study design was observational study. The studies were included if full text is available. Studies were excluded if they met the following criteria: (1) Studies if the patients had pre-existing sleep disorders or sleep apnea or breathing problem. (2) Studies conducted in populations other than Asia were excluded. (3) The conference abstracts, and letter to the editor were excluded due to lack of detailed information.

\subsection{Data extraction}

Two independent authors (MA and MA) performed study screening and data extraction judiciously according to inclusion criteria. Any discrepancies were resolved through discussion of the third senior authors (PG, PK and MS) until consent is achieved on every issue. A standard data extraction format was used to collect the study information including; name of the first author, year of the publication, country, inclusion criteria, participants, interventions, study design, sample size, mean age, percentage of male, population source, mean body mass index (BMI) $\mathrm{kg} / \mathrm{m},{ }^{2}$ mean $\mathrm{HbA1c} \%$, mean FPG $\mathrm{mg} / \mathrm{dl}$, duration of diabetes (year), sleep measure assessment.

\subsection{Quality assessment}

Two authors (MA and MA) independently assessed the quality of the methodology for each observational studies by using the Downs and Black assessment tool. ${ }^{16}$ All disagreements were resolved by discussion of the third senior reviewer adjudication (PG, PK and MS).

The assessment tool comprised of 26 items categorized into five subscales: reporting ( 9 items items), external validity (3 items), Bias (7 items), confounding ( 6 items), and power ( 1 item). The Downs and Black assessment tool, answer were generally scored as 0 or 1 , except for one item in the reporting subscale, which scored as 0 to 2 and the single item on power, which was scored as 0 to 5 . Therefore, the total maximum score was assigns 31 .

\subsection{Data synthesis and statistical analysis}

To examine the relationship between sleep duration, sleep quality and diabetes. Duration of sleep was categorized into three categories; short, long and reference group for each study. Similarly, the sleep quality also categorized into poor sleep and good sleep. Pooled mean difference (MD) and 95\% confidence intervals (CIs) was estimated using a weighted random-effect model.

We tested for clinical heterogeneity inherent in our data, by using an inverse variance-weighted random effects models (DerSimonian and Laird) for meta-analyses. ${ }^{17}$

In addition, we compared the reference category for each study with good and poor sleep quality respectively and estimated the mean difference using the random effects model. Statistical heterogeneity examined by using the $\mathrm{I}^{2}$ statistics and Cochran's $\mathrm{Q}$ test with results ranging from 0 to $100 \%$ and values of 25,50 and $75 \%$ representing low, moderate and high levels of heterogeneity, respectively. ${ }^{18}$

The potential publication bias was assessed by visual inspection of the funnel plot for asymmetry and was examined by using Egger's linear regression test. ${ }^{19}$

The subgroup analysis was carried out, to measure the possible sources of heterogeneity and determine the potential impact of sleep duration or sleep quality on HbA1c and FPG respectively, with compared to reference category used for sleep. All statistical analysis was performed using the Cochrane's Collaboration RevMan (Version 5.3). The data were considered statistically significant when $p<0.05$.

\section{Results}

\subsection{Literature search and study inclusion}

The PRISMA flow chart summarizing the process of study selection is shown in Fig. 1.

The two electronic databases; PubMed $(n=1187)$ and Embase ( $n=673$ ) search identified 1860 potentially relevant records, of which 1740 studies remained after removing duplicate records. 1485 were 

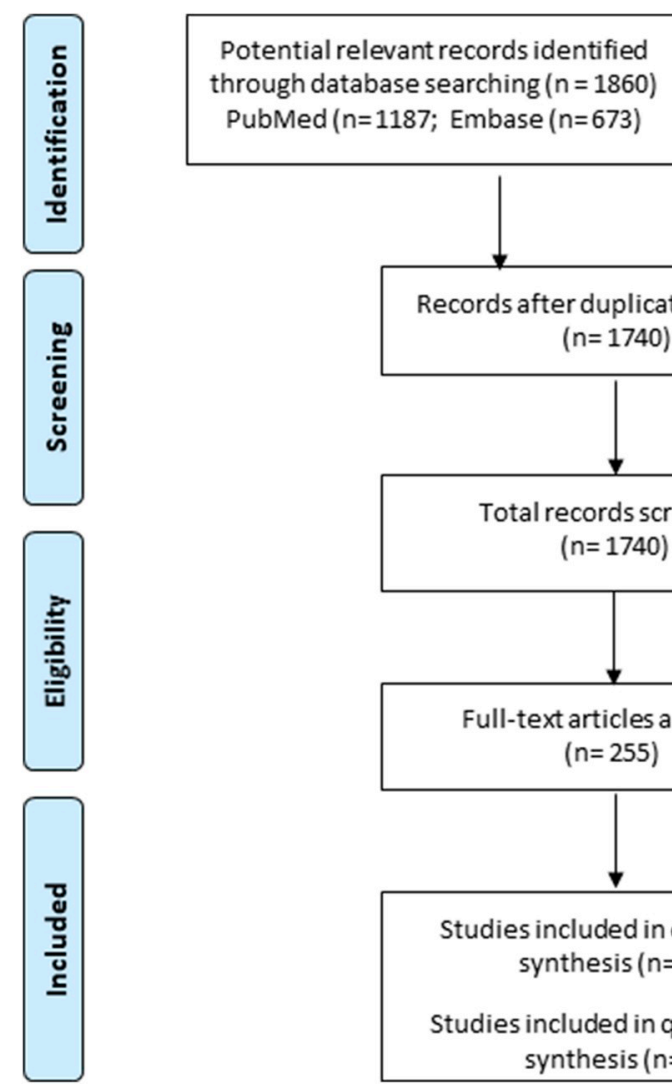

Additional records identified through

other sources

$(n=0)$

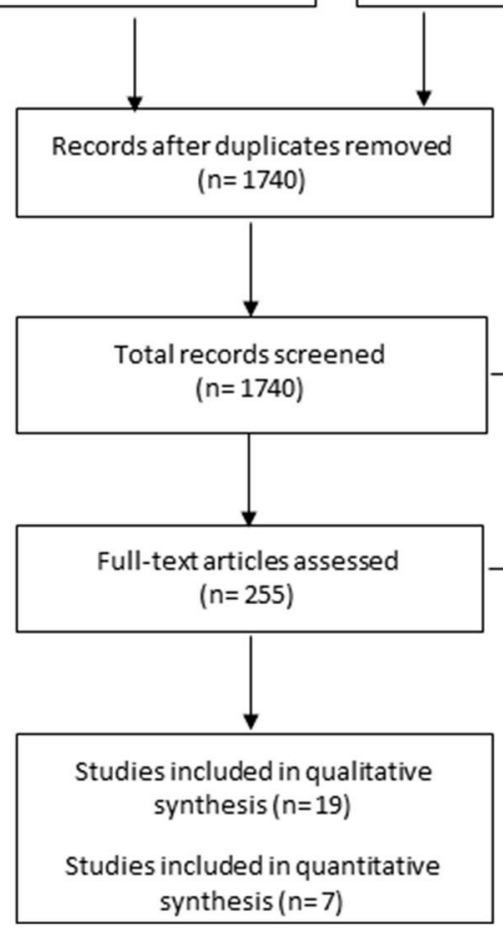

Records excluded on the basis of title

and abstract $(n=1485)$

Full-text articles excluded, with reasons $(n=236)$

Review $(n=68)$, Conference abstract $(n=$ 19), Animal study $(n=84)$ Population not of interest $(n=42)$, Outcome not of interest $(n=23)$

Fig. 1. PRISMA flow diagram of study selection process.

excluded after screening the title and abstract on the basis of inclusion and exclusion criteria. The remaining 225 full-text articles were assessed for eligibility, and 236 were further excluded due to review ( $\mathrm{n}=68)$, conference abstract $(\mathrm{n}=19)$, animal study $(\mathrm{n}=84)$, population not of interest $(n=42)$, outcome not of interest $(n=23)$. Total 19 articles were included in the systematic literature review, further 12 studies were not included in meta-analysis due to data were not suitable for meta-analysis. Finally, seven studies were included in the meta-analysis.

\subsection{Study characteristics and quality assessment}

The summary study characteristics of included studies are presented in Table 1. Total 19 observational studies included in the systematic literature review. Of 19 studies, seven studies were eligible for the meta-analysis. The qualitative studies included 74,887 participants with mean age ranged from 42.2 to 66.0 years. These studies were conducted in China $(\mathrm{n}=5),{ }^{20-24}$ Japan $(\mathrm{n}=4),{ }^{25-28}$ South Korea $(\mathrm{n}=2)^{29,30}$ Taiwan $(\mathrm{n}=2)^{31,32}$ and one each in India, ${ }^{33}$ Iran, ${ }^{34}$ Thailand, ${ }^{35}$ Turkey, ${ }^{36}$ Singapore, ${ }^{37}$ UAE. ${ }^{38}$ The mean HbA1c levels, BMI $(\mathrm{kg} / \mathrm{m} 2)$, and FPG $(\mathrm{mg} / \mathrm{dL})$ were ranged from 7.0 to $8.52 \%, 23.8$ to 29.5 , and 108.77 to 168.00 respectively. The methodological quality assessment of the included studies is depicted in supplementary Table S2. The included studies in this review scored from 10 to 20 (out of 31) on the risk of bias assessment tool. Majority of the studies scored (15-20) suggesting the high quality. On reporting and selection of bias, all the studies scored an average or above average.

\subsection{Sleep duration}

The sleep duration was examined by using either a single survey item or from the Pittsburg Sleep Quality Index (PSQI) questionnaire across the included studies. There was diverse definition mentioned for normal sleep duration, the common reference category for normal sleep duration was $6-8 \mathrm{~h}$ of sleep per night, ${ }^{20} 6.5-7.4 \mathrm{~h},{ }^{25} 6-7.9 \mathrm{~h},{ }^{23}$ and $7 \mathrm{~h}$ of sleep ${ }^{30}$ per night as the reference category.

Majority of studies ( $\mathrm{n}=3$ ) defining short sleep duration as $<6 \mathrm{~h}$ per night, ${ }^{20,23,30}$ while other studies defined as either $<4.5 \mathrm{~h} .{ }^{25}$ Whereas, one study reported long sleep duration which was defined as $>8 \mathrm{~h}$ per night, ${ }^{20}$ one study as $>9 \mathrm{~h},{ }^{30}$ one study as $>8.5 \mathrm{~h}^{25}$ and one study in $>9 \mathrm{~h} .{ }^{23}$ The definition of sleep duration and sleep quality for included studies were detailed in supplementary Table S1.

Meta-analysis was performed on the basis of duration of sleep and impact of HbA1c levels and FPG. The sleep duration and association of HbA1c levels were reported in four studies. ${ }^{23,27,30,37}$ In addition, three studies reported $^{23,27,30}$ sleep duration and association with the FPG.

From the included studies, the association between short and long sleep was potentially associated with higher levels of HbA1c, ${ }^{23,25,30}$ while the data from other studies shown that short sleep duration was significantly associated with higher levels of HbA1c. ${ }^{34,35}$

\subsection{Short duration of sleep}

The total four studies ${ }^{23,27,30,37}$ with 28,105 participants were included in the meta-analysis involving 4006 participants examining the effects of sleep duration on HbA1c control compared to normal sleep duration ( $\mathrm{n}=24099)$. Short sleep was significantly associated with higher HbA1c levels (mean difference (MD: 0.11; 95\% CI: 0.06-0.17), when compared to normal sleep, forest plot shown in Fig. 2a. There was no such evidence of statistical heterogeneity $\left(\mathrm{Q}=0.81, \mathrm{I}^{2}=0 \%\right.$, $\mathrm{p}=0.85$ ). While, there was no statistical significant difference was found between short sleep duration and higher level of FPG (MD: 2.28; 95\% CI: 0.64-5.20) compared to normal sleep (Fig. 2b). The shape of funnel plots for all studies suggests that there was no such evidence of publication bias (Egger's test, $\mathrm{p}=0.09$ ). 


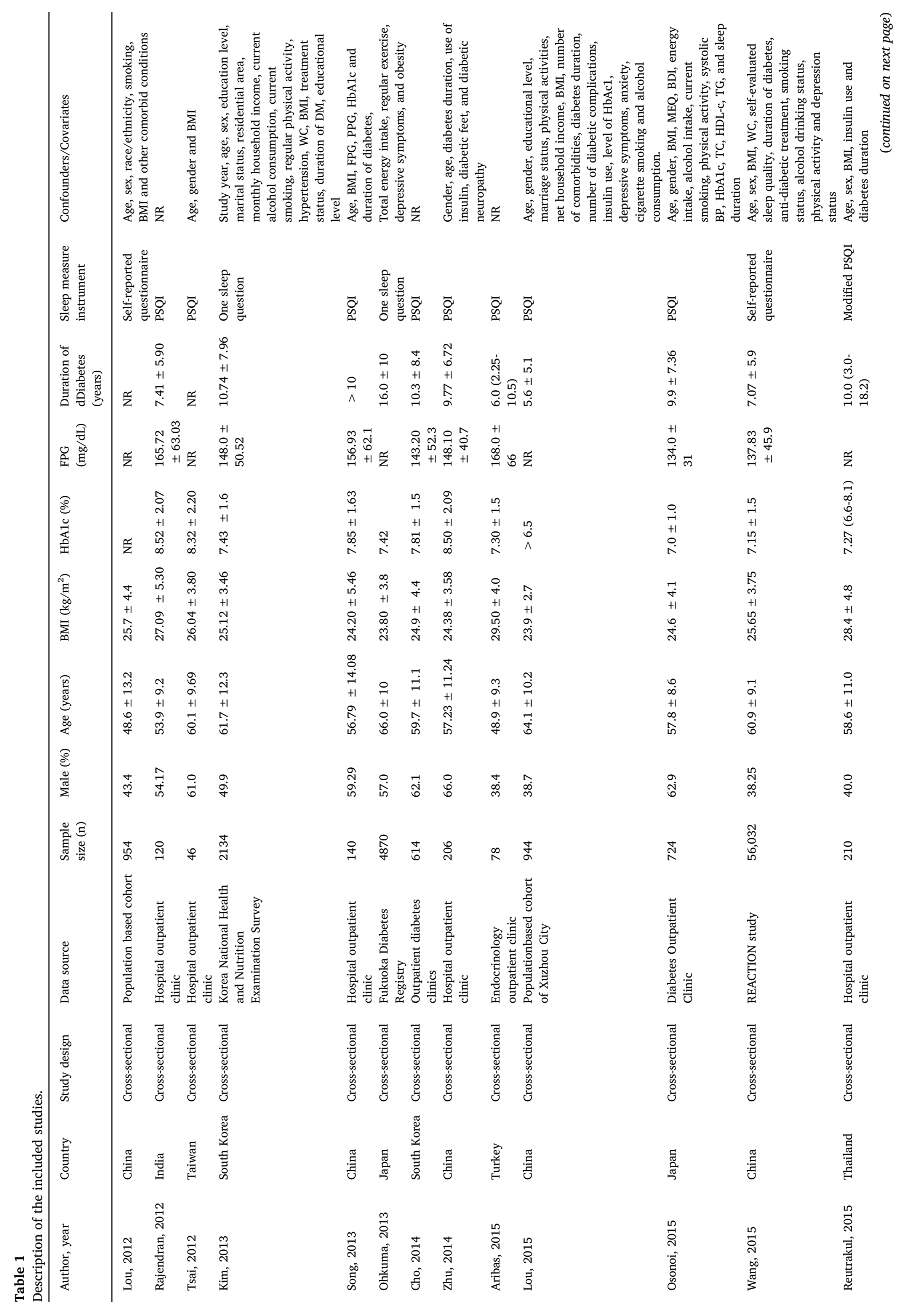




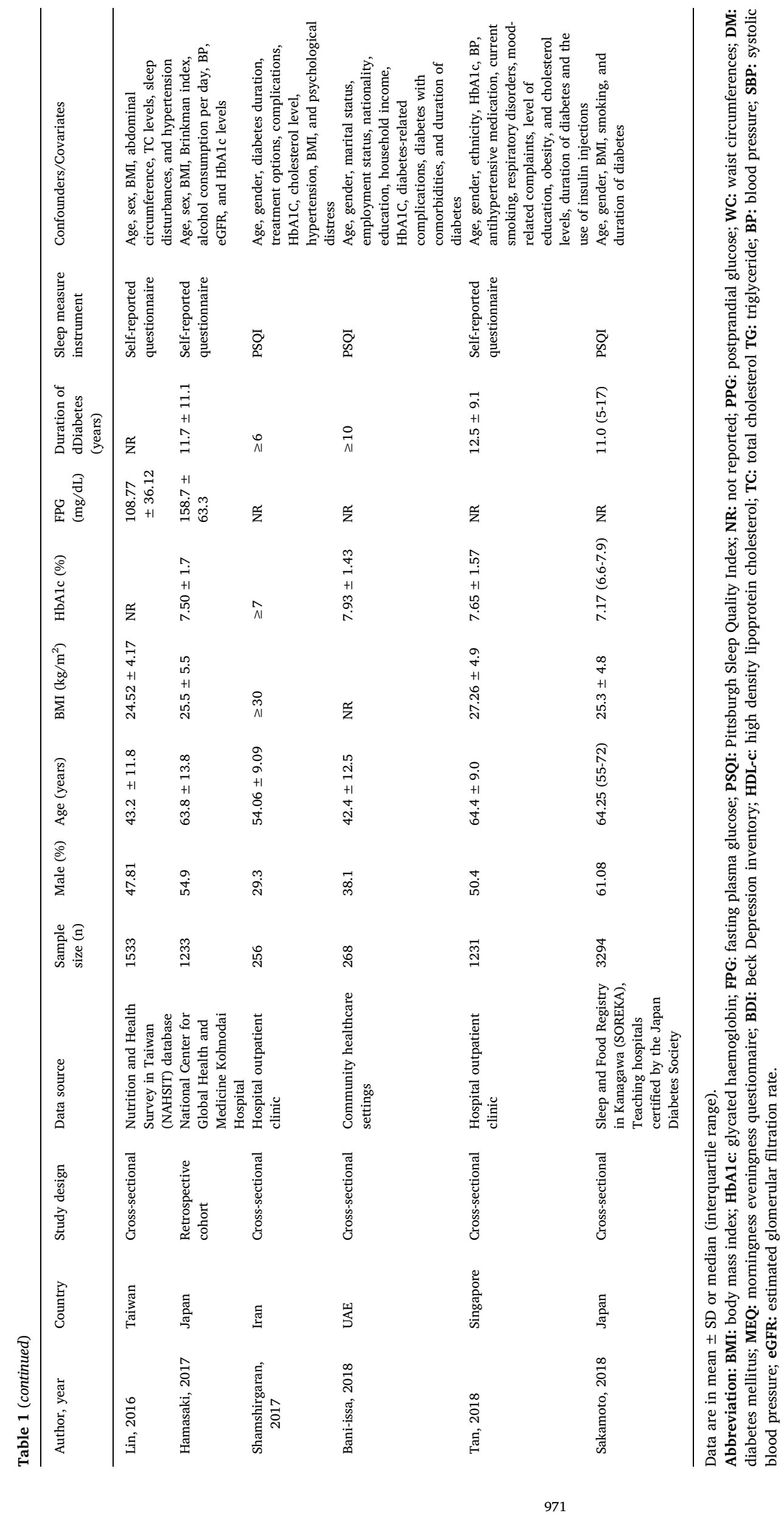


a. Short sleep duration and HbA1c levels

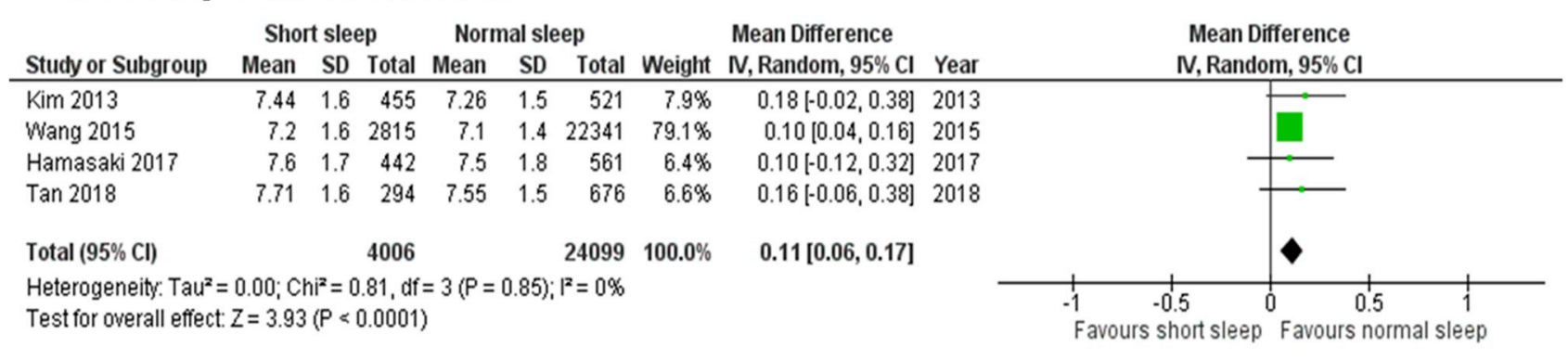

b. Short sleep duration and FPG levels

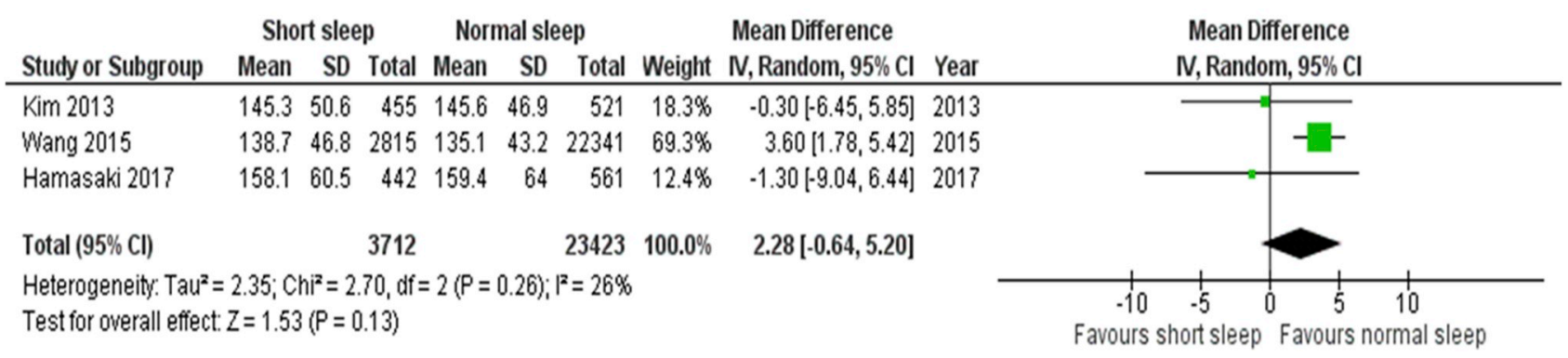

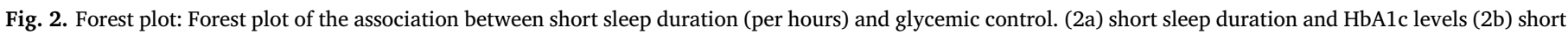
sleep duration and FPG levels.

\subsection{Long duration of sleep}

There was no significant difference was found between long sleep duration and HbA1c levels (MD: 0.12; 95\% CI: 0.06-0.30) shown in Fig. 3a. There was evidence of moderate statistical heterogeneity $\left(\mathrm{Q}=11.12, \mathrm{I}^{2}=73 \%, \mathrm{p}=0.01\right)$. However, long sleep duration was significantly associated with higher FPG (MD: 5.30; 95\% CI: 3.27-7.34) compared to normal sleep duration (Fig. 3b). The examination of funnel plot symmetry for studies assessing impact of long sleep duration on
HbA1c control was reported no evidence of publication bias (Egger's test, $\mathrm{p}=0.07)$.

\subsection{Sleep quality}

Three studies ${ }^{24,26,36}$ included in this meta-analysis which examined the effects of sleep quality on HbA1c levels and FPG. The Pittsburgh Sleep Quality Index (PSQI) instrument was used to determine the sleep quality across the included studies. There is various definition of poor

\section{a. Long sleep duration and HbA1c levels}

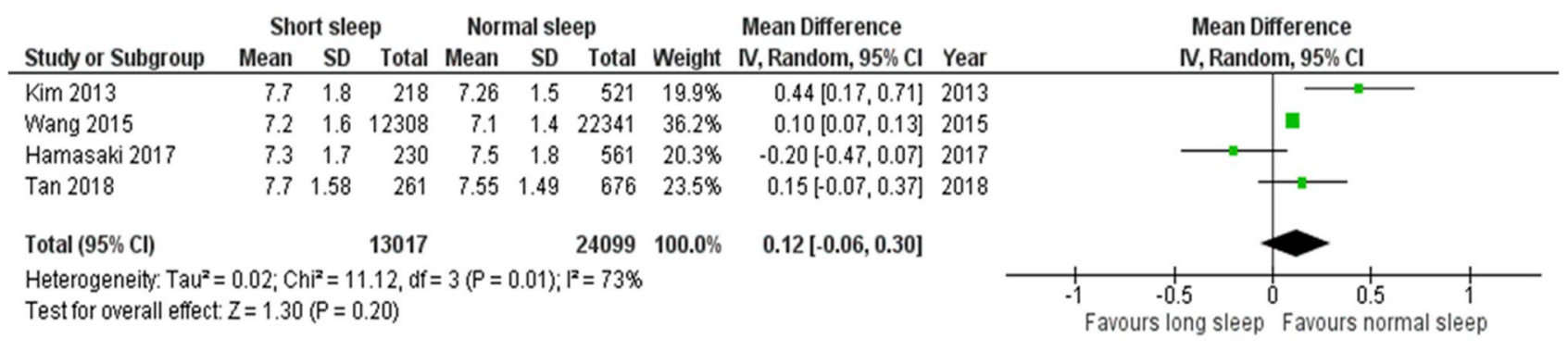

b. Long sleep duration and FPG levels

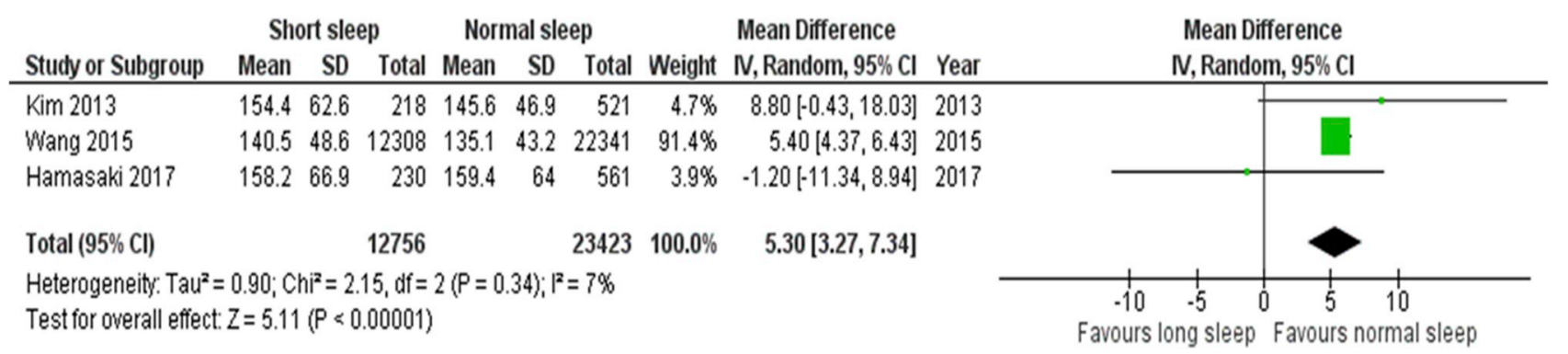

Fig. 3. Forest plot: Forest plot of the association between long sleep duration (per hours) and glycemic control (3a) long sleep duration and HbA1c levels (3b) long sleep duration and FPG levels. 


\section{a. Sleep quality and HbA1c levels}

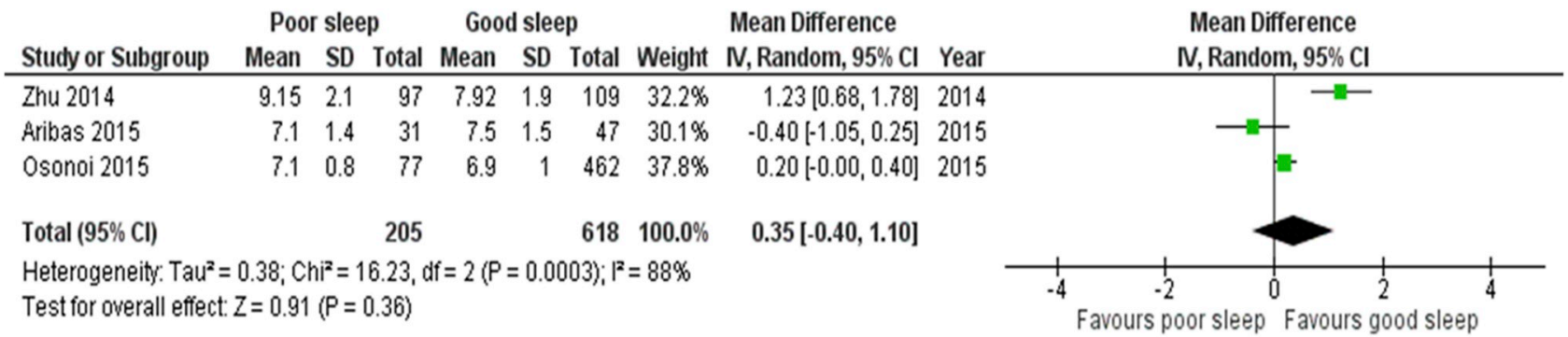

\section{b. Sleep quality and FPG levels}

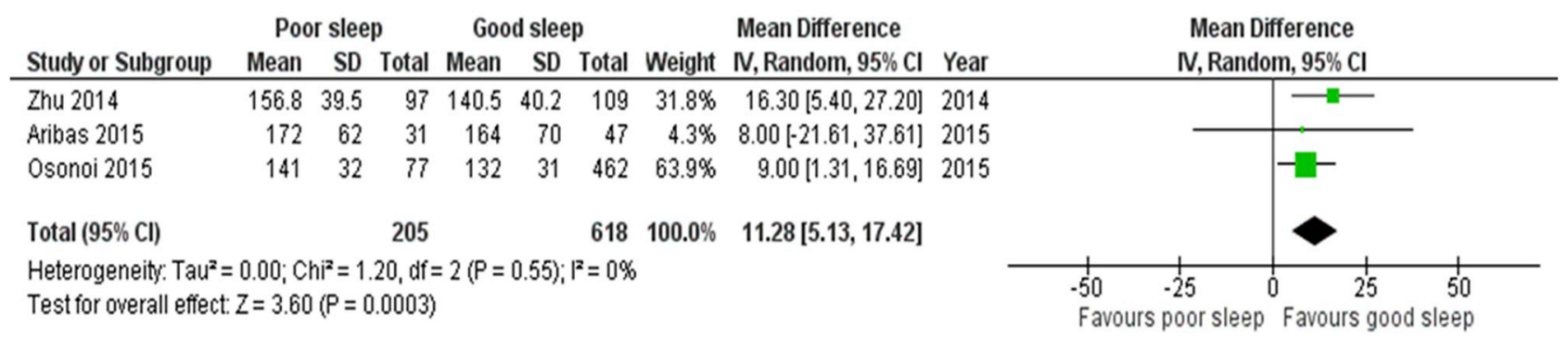

Fig. 4. Forest plot: Forest plot of the association between sleep quality and glycemic control (4a) sleep quality and HbA1c levels (4b) sleep quality and FPG levelsGreen

squares and horizontal lines represent study-specific estimates and $95 \%$ CI. The size of the square is proportional to the weights of the individual studies. Black diamond represents pooled estimates (center) and 95\% CI (width).

sleep quality reported in the included study. According to PSQI global score, the poor sleep quality ranged with 5 to $\geq 9$. Majority of studies ( $\mathrm{n}=6$ ) reported PSQI global score $\geq 5,{ }^{22,29,33,35,36,38}$ one study as $>7,{ }^{21}$ two study $\geq 8,{ }^{24,31}$ and one study $\geq 9 .{ }^{26}$ Evidence from included studies shown the poor sleep quality significantly associated with poor glycemic control. ${ }^{24,31}$

After adjusting for gender, age, diabetes duration, use of insulin, diabetic feet, and diabetic neuropathy, the adjusted model shows sleep disturbance (OR: 5.09; 95\% CI: 1.48-17.52), sleep latency (OR: 2.14; 95\% CI: 1.26-3.69) and daytime dysfunction (OR: 3.50; 95\% CI: 2.02-6.07) were significantly associated with higher HbA1c levels. ${ }^{24}$ Similarly, a study performed by Osonoi and colleagues reported that higher HbA1c and fasting glucose levels were significantly associated with poor sleep quality, after adjusting age and sex. ${ }^{26}$

Results from meta-analysis shown that the good sleep quality was significantly associated with lowering of FPG (MD: 11.28; 95\% CI: 5.13-17.42) shown in Fig. 4b. Whereas, the good sleep quality did not show any significant difference in HbA1c levels when compared to poor sleep quality (MD: 0.35; 95\% CI: 0.40.1.10) shown in Fig. 4a. The shape of funnel plots for all studies suggested, there was no such evidence of publication bias (Egger's test: $\mathrm{p}=0.17$ ). The higher statistical heterogeneity was found $\left(\mathrm{Q}=16.23, \mathrm{I}^{2}=88 \%, \mathrm{p}<0.001\right)$.

\section{Discussion}

To the best of our knowledge, this is the first systematic literature review and meta-analysis that portrayed the evidence for an association between sleep duration, sleep quality and glycaemic control among T2DM patients in Asian countries. This study will, expectantly, bring attention of health practitioner to examine the effect of sleep quality in improving glycaemic control, and importance of reducing the worsen of disease conditions most densely populated share of the Asia like China and India. The summary results from our studies suggest that short sleep was significantly associated with higher HbA1c levels, suggesting poorer glycemic control, when compared to normal sleep duration. However, good sleep quality was significantly associated with lowering of FPG levels compared to poor sleep quality among T2DM patients.

Several meta-analyses examined the association between sleep duration and obesity, ${ }^{6}$ hypertension ${ }^{7}$ and type 2 diabetes. ${ }^{8}$ Since, obesity and impaired fasting glucose are major components of incidence of diabetes, it is obvious that short sleep duration was significantly associated with poor glycemic control. Although, recent published metaanalysis established the association between sleep duration, sleep quality and glycemic control among T2DM patients. ${ }^{4}$

The potential mechanism underlying the association between sleep and glycemic control among diabetic patients are yet not well documented.

While the results from experimental studies as well as epidemiological studies suggested that sleep deprivation is associated with insulin resistance, increased appetite and impaired glucose tolerance in healthy subjects. ${ }^{39,40}$ Insufficient sleep leads to disturb energy balance via upregulation of hunger and appetite.

Similarly, evidence from a study reported that, frequent sleep impairment and short sleep duration associated with significantly lower levels of leptin and higher level of ghrelin, as these both hormones play important role to regulate satiety and appetite. ${ }^{41}$

Additionally, nocturnal sleep loss and awakening has been associated with reduced leptin levels and leptin resistance which may lead to dysregulation of the hypothalamic-pituitary-adrenal axis, resulting in impaired insulin sensitivity and glucose impairment which may affect glycaemic control. ${ }^{42}$

Results from our studies shown that sleep alteration is associated with higher HbA1c levels. The results of this study is in line with other epidemiological studies which noted that poor sleep have detrimental impact on health facets including glycemic control, ${ }^{11}$ increased incidence of coronary artery calcification ${ }^{43}$ and hypertension. ${ }^{7}$ Several meta-analyses examined the impact of sleep duration and the risk of $\mathrm{T} 2 \mathrm{DM}$, indicated that short or long sleep duration was significantly 
associated with increased incidence of T2DM. ${ }^{8,11,44}$ Consistently our study also confirmed that short sleep duration and long sleep duration significantly associated with poor glycemic control and higher FPG levels respectively. There was a $0.11 \%$ increase in HbA1c levels in patients who has short sleep durations of $<4.5-6 \mathrm{~h} /$ night and $5.30 \%$ increase in FPG levels in those with long sleep duration of $\geq 8 \mathrm{~h}$.

A study conducted by Chasen and colleagues, investigating association of impaired sleep quality and daytime sleepiness of 107 T2DM patients. The study suggested poor sleep quality was significantly associated with difficulty of multiple aspects of health services such as self-care adherence, medication adherence, and dietary adherence which leads to poorer glycemic control. ${ }^{45}$ Data from the study conducted by Sundaram et al. documented that higher HbA1c levels was associated with emotional distress in patients with T2DM. ${ }^{46}$ The concluded evidence from these studies observed, that by affecting the personal self-care activities, including diet and exercise, ${ }^{45}$ resulted into poor glycaemic control.

Studies assessing potential impact of sleep quality on metabolic outcomes. A study conducted by Jike et al. examined the association between long sleep duration and multiple health outcomes, the results from meta regression found statistically significant associations between longer sleep duration and increased mortality rate and incident of CVD disease. ${ }^{44}$

The epidemiological study suggested patients with obstructive sleep apnea (OSA), significantly associated with obese T2DM patients having poor glycemic control. Results from multinational study European Sleep Apnea Cohort $(\mathrm{n}=6616)$ has shown that the adjusted mean HbA1c levels were higher $(0.72 \%)$ in patients with severe OSA when compared with patients without OSA, ${ }^{47}$ confirmed that comorbid conditions play as a confounding factor in this association.

Evidence from the study suggested that sleep hygiene in addition with smoking and obesity is an important modifiable risk factor for improving glycaemic control in patients with T2DM. ${ }^{48}$

Recently, a meta-analysis conducted by Lee and colleagues evaluated the relationship between glycemic control in patients with T2DM and the amount of sleep as well as quality of sleep and reported that short and long sleep durations were associated with an increased level of HbA1c (weighted mean difference (WMD): $0.23 \%$ [0.10-0.36], and WMD: $0.13 \%$ [0.02-0.25] respectively compared to normal sleep. Additionally, poor sleep quality was also associated with a higher level of HbA1c (WMD: $0.35 \%$ [0.12-0.58]). ${ }^{4}$ However, the current metaanalysis examined the effect of sleep duration as well sleep quality on glycemic control among Asian T2DM patients included 6 studies for quantitative analysis, observed that short sleep was significantly associated with higher HbA1c levels, while long sleep duration was significantly associated with higher FPG levels. Similarly, good sleep quality was significantly reducing the FPG levels but did not show any significant reduction in HbA1c level. Therefore, results from our study suggests that satisfactory sleep could play an important role in regards to glycemic control in patients with T2DM.

The complete prevention of impaired sleep across the globe is unattainable, while increased knowledge about the physiological importance of this condition, can improved sleep schedule or even working schedule for shift workers possibly will developed.

Additionally, with the improvement of sleep hygiene and sleep quality that could enhance the body's restorative function to maintain glucose homeostasis which avoid altering the glucose metabolism.

Furthermore, the knowledge and awareness of patients towards importance of sleep health that may play a crucial approach to help to improve glycaemic control in T2DM patients. The extensive implementation of this approach, could be a betterment for T2DM patients and important public health consequences. As a data from the United Kingdom Prospective Diabetes Study (UKPDS) reported that for every $1 \%$ reduction in mean $\mathrm{HbA1c}$, this would translate to a $21 \%$ reduction in death, $14 \%$ reduction in myocardial infarction and $37 \%$ decrease in microvascular complications at population level. ${ }^{49}$ Our findings also contribute the consistent results with $0.11 \%$ reduction in mean HbA1c levels those who have normal sleep duration.

\subsection{Strength}

Our study has several strengths. First, to our best of knowledge, this is the first study assessed the impact of sleep duration and sleep quality on glycemic control among T2DM patients in the Asian population. Second, we explored sources of heterogeneity with subgroup analysis on the basis of short or long sleep duration compared to reference group, as well as poor and good sleep quality compared to reference group. Lastly, the presence of heterogeneity in this meta-analysis minimally affected our results.

\subsection{Limitations}

There are certain limitations in our study that merit further discussion. First, we could not have examined the sensitivity analysis due to minimal number of studies qualified for meta-analysis.

Second, we could not have explored the impact of sleep quality and sleep duration on glycemic control in regard to population source, age group, BMI, due to the incomplete information in maximum of included studies. These factors (age, BMI and others) may be considered as a confounder which may alter the results in order to under or overestimation of the association.

Third, variation in the definition of sleep duration among the included studies. Our study relies on the definition used in individual observational studies. The different measurement approaches and varying definition of sleep duration might lead to differences among the included studies.

Fourth, majority of included studies were cross sectional study design, which may not allow for causal inference. Fifth, all included studies measured sleep duration using subjective questionnaires or an interview question rather than objective measures such as actigraphy or polysomnography. Nevertheless, measurement of sleep duration based on a self-reported questionnaire, simple question, sleep diary, and objective measures have been reported inconclusive results. ${ }^{42,43}$ Lastly, as sleep duration and quality of sleep were examined at single time point in all studies, this might not satisfactorily capture the impact of sleep disruption in patients with T2DM. Therefore, alteration of sleep patterns and glycaemic control over time could be a better consideration of the actual impact of the exposure.

\section{Conclusions}

In conclusion, findings from our study suggests that short sleep was significantly associated with higher HbA1c levels or poorer glycaemic control, whereas, good sleep quality was significantly reducing the FPG levels. Therefore, sleep duration as well as sleep quality may be consider as a novel predictors and independent risk factor for estimation of poorer glycaemic control in patients with T2DM. However, further well designed prospective studies will be warranted to establish the potential association between sleep and glycemic control.

\section{Ethical approval}

This article does not contain any studies with human participants or animals performed by any of the authors.

\section{Declaration of competing interest}

There is no known conflict of interest associated with this publication. 


\section{Acknowledgements}

First author (MA) thankful to Sun Pharmaceutical, India, for providing assistantship for this project under the joint collaboration for the Ph.D. programme with Jamia Hamdard, New Delhi, India.

\section{Appendix A. Supplementary data}

Supplementary data to this article can be found online at https:// doi.org/10.1016/j.cegh.2020.03.006.

\section{References}

1. Zimmet PZ, Magliano DJ, Herman WH, Shaw JE. Diabetes: a 21st century challenge. Lancet Diabetes Endocrinol. 2014 Jan 1;2(1):56-64.

2. International Diabetes Federation. IDF Diabetes Atlas. eighth ed. Brussels, Belgium: International Diabetes Federation; 2017.

3. Haffner SM. Coronary heart disease in patients with diabetes. $N$ Engl J Med. 2000;342:1040-1042.

4. Lee SW, Ng KY, Chin WK. The impact of sleep amount and sleep quality on glycemic control in type 2 diabetes: a systematic review and meta-analysis. Sleep Med Rev. 2017 Feb 1;31:91-101.

5. National sleep foundation. Foundation NS. 2018 sleep in America poll. Washington DC. https://www.sleepfoundation.org/; 2018 Accessed on 01 June 2019.

6. Cappuccio FP, Taggart FM, Kandala NB, et al. Meta-analysis of short sleep duration and obesity in children and adults. Sleep. 2008 May 1:31(5):619-626.

7. Wang Q, Xi B, Liu M, Zhang Y, Fu M. Short sleep duration is associated with hypertension risk among adults: a systematic review and meta-analysis. Hypertens Res. 2012 Oct;35(10):1012.

8. Cappuccio FP, D'Elia L, Strazzullo P, Miller MA. Quantity and quality of sleep and incidence of type 2 diabetes: a systematic review and meta-analysis. Diabetes Care. 2010 Feb 1;33(2):414-420.

9. Cappuccio FP, Cooper D, D'Elia L, Strazzullo P, Miller MA. Sleep duration predicts cardiovascular outcomes: a systematic review and meta-analysis of prospective studies. Eur Heart J. 2011 Feb 7;32(12):1484-1492.

10. Cappuccio FP, D'Elia L, Strazzullo P, Miller MA. Sleep duration and all-cause mortality: a systematic review and meta-analysis of prospective studies. Sleep. 2010 May 1;33(5):585-592.

11. Knutson KL, Ryden AM, Mander BA, Van Cauter E. Role of sleep duration and quality in the risk and severity of type 2 diabetes mellitus. Arch Intern Med. 2006 Sep 18;166(16):1768-1774

12. Schmid SM, Hallschmid M, Schultes B. The metabolic burden of sleep loss. Lancet Diabetes Endocrinol. 2015 Jan 1;3(1):52-62.

13. Miller MA, Cappuccio FP. Inflammation, sleep, obesity and cardiovascular disease. Curr Vasc Pharmacol. 2007 Apr 1;5(2):93-102.

14. Buxton OM, Marcelli E. Short and long sleep are positively associated with obesity, diabetes, hypertension, and cardiovascular disease among adults in the United States. Soc Sci Med. 2010 Sep 1;71(5):1027-1036.

15. Moher D, Liberati A, Tetzlaff J, Altman DG. PRISMA group the PRISMA group preferred reporting items for systematic reviews and meta-analyses. Prisma Statement BMJ. 2009;339:b2535.

16. Downs SH, Black N. The feasibility of creating a checklist for the assessment of the methodological quality both of randomised and non-randomised studies of health care interventions. J Epidemiol Community Health. 1998 Jun 1;52(6):377-384.

17. DerSimonian R, Laird N. Meta-analysis in clinical trials. Contr Clin Trials. 1986 Sep $1 ; 7(3): 177-188$.

18. Higgins JP, Green S. Cochrane Handbook for Systematic Reviews of Interventions. The Cochrane Collaboration; 2011 Version 5.1.0 [updated March 2011].

19. Egger M, Smith GD, Schneider M, Minder C. Bias in meta-analysis detected by a simple, graphical test. BMJ. 1997 Sep 13;315(7109):629-634.

20. Lou P, Chen P, Zhang L, et al. Relation of sleep quality and sleep duration to type 2 diabetes: a population-based cross-sectional survey. BMJ open. 2012 Jan 1;2(4):e000956.

21. Lou P, Qin Y, Zhang P, et al. Association of sleep quality and quality of life in type 2 diabetes mellitus: a cross-sectional study in China. Diabetes Res Clin Pract. 2015 Jan 1;107(1):69-76.

22. Song Y, Ye X, Ye L, Li B, Wang L, Hua Y. Disturbed subjective sleep in Chinese females with type 2 diabetes on insulin therapy. PloS One. 2013 Jan 28;8(1):e54951.

23. Wang $\mathrm{T}$, Lu J, Wang $\mathrm{W}$, et al. Sleep duration and snoring associate with hypertension and glycaemic control in patients with diabetes. Diabet Med. 2015 Aug;32(8):1001-1007.

24. Zhu BQ, Li XM, Wang D, Yu XF. Sleep quality and its impact on glycaemic control in patients with type 2 diabetes mellitus. Int J Nurs Sci. 2014 Sep 1;1(3):260-265.

25. Ohkuma T, Fujii H, Iwase M, et al. Impact of sleep duration on obesity and the glycemic level in patients with type 2 diabetes: the Fukuoka Diabetes Registry. Diabetes Care. 2013 Mar 1;36(3):611-617.

26. Osonoi Y, Mita T, Osonoi T, et al. Poor sleep quality is associated with increased arterial stiffness in Japanese patients with type 2 diabetes mellitus. BMC Endocr Disord. 2015 Dec;15(1):29.

27. Hamasaki H, Katsuyama H, Sako A, Yanai H. Short sleep duration is associated with B-type natriuretic peptide levels and predicts the death of Japanese patients with type 2 diabetes. Sleep Med. 2017 Aug 1;36:1-5.

28. Sakamoto R, Yamakawa T, Takahashi K, et al. Association of usual sleep quality and glycemic control in type 2 diabetes in Japanese: a cross sectional study. Sleep and Food Registry in Kanagawa (SOREKA). PloS One. 2018 Jan 24;13(1):e0191771.

29. Cho EH, Lee H, Ryu OH, Choi MG, Kim SW. Sleep disturbances and glucoregulation in patients with type 2 diabetes. J Kor Med Sci. 2014 Feb 1;29(2):243-247.

30. Kim BK, Kim BS, An SY, et al. Sleep duration and glycemic control in patients with diabetes mellitus: Korea national health and nutrition examination survey 20072010. J Kor Med Sci. 2013 Sep 1;28(9):1334-1339.

31. Tsai YW, Kann NH, Tung TH, et al. Impact of subjective sleep quality on glycemic control in type 2 diabetes mellitus. Fam Pract. 2011 Jul 27;29(1):30-35.

32. Lin CL, Tsai YH, Yeh MC. Associations between sleep duration and type 2 diabetes in Taiwanese adults: a population-based study. J Formos Med Assoc. 2016 Sep 1;115(9):779-785.

33. Rajendran A, Parthsarathy S, Tamilselvan B, Seshadri KG, Shuaib M. Prevalence and correlates of disordered sleep in southeast asian indians with type 2 diabetes. Diabetes Metabol. J. 2012 Feb 1;36(1):70-76.

34. Shamshirgaran SM, Ataei J, Malek A, Iranparvar-Alamdari M, Aminisani N. Quality of sleep and its determinants among people with type 2 diabetes mellitus in Northwest of Iran. World J Diabetes. 2017 Jul 15;8(7):358.

35. Reutrakul S, Siwasaranond N, Nimitphong H, et al. Relationships among sleep timing, sleep duration and glycemic control in Type 2 diabetes in Thailand. Chronobiol Int. 2015 Nov 26;32(10):1469-1476.

36. Aribas A, Kayrak M, Tekinalp M, et al. The relationship between serum asymmetric dimethylarginine levels and subjective sleep quality in normotensive patients with type 2 diabetes mellitus. Kor J Intern Med. 2015 May;30(3):316.

37. Tan NY, Chew M, Tham YC, et al. Associations between sleep duration, sleep quality and diabetic retinopathy. PloS One. 2018 May 24;13(5):e0196399.

38. Bani-issa W, Al-Shujairi AM, Patrick L. Association between quality of sleep and health-related quality of life in persons with diabetes mellitus type $2 . J$ Clin Nurs. 2018 Apr;27(7-8):1653-1661.

39. Spiegel K, Leproult R, Van Cauter E. Impact of sleep debt on metabolic and endocrine function. Lancet. 1999 Oct 23;354(9188):1435-1439.

40. Stamatakis KA, Punjabi NM. Effects of sleep fragmentation on glucose metabolism in normal subjects. Chest. 2010 Jan 1;137(1):95-101.

41. Taheri S, Lin L, Austin D, Young T, Mignot E. Short sleep duration is associated with reduced leptin, elevated ghrelin, and increased body mass index. PLoS Med. 2004 Dec 7;1(3):e62.

42. Pejovic S, Vgontzas AN, Basta M, et al. Leptin and hunger levels in young healthy adults after one night of sleep loss. J Sleep Res. 2010 Dec;19(4):552-558.

43. King CR, Knutson KL, Rathouz PJ, Sidney S, Liu K, Lauderdale DS. Short sleep duration and incident coronary artery calcification. Jama. 2008 Dec 24;300(24):2859-2866.

44. Jike M, Itani O, Watanabe N, Buysse DJ, Kaneita Y. Long sleep duration and health outcomes: a systematic review, meta-analysis and meta-regression. Sleep Med Rev. 2018 Jun 1;39:25-36.

45. Chasens ER, Korytkowski M, Sereika SM, Burke LE. Effect of poor sleep quality and excessive daytime sleepiness on factors associated with diabetes self-management. Diabetes Educat. 2013 Jan;39(1):74-82.

46. Sundaram M, Kavookjian J, Patrick JH, Miller LA, Madhavan SS, Scott VG. Quality of life, health status and clinical outcomes in Type 2 diabetes patients. Qual Life Res. 2007 Mar 1;16(2):165-177.

47. Kent BD, Grote L, Ryan S, et al. Diabetes mellitus prevalence and control in sleepdisordered breathing: the European Sleep Apnea Cohort (ESADA) study. Chest. 2014 Oct 1;146(4):982-990.

48. Wannamethee SG, Shaper AG, Perry IJ. Smoking as a modifiable risk factor for type 2 diabetes in middle-aged men. Diabetes Care. 2001 Sep 1;24(9):1590-1595.

49. Stratton IM, Adler AI, Neil HA, et al. Association of glycaemia with macrovascular and microvascular complications of type 2 diabetes (UKPDS 35): prospective observational study. BMJ. 2000 Aug 12;321(7258):405-412. 